\title{
In pursuit of human dignity
}

\author{
David Badcott • Carlo Leget
}

Published online: 26 September 2013

(C) Springer Science+Business Media Dordrecht 2013

\begin{abstract}
...the march of scientific progress that now promises to give us manipulative power over human nature itself ... will eventually compel us to take a stand on the meaning of human dignity, understood as the essential and inviolable core of our humanity.
\end{abstract}

Human Dignity and Bioethics, Essays Commissioned by the President's Council on Bioethics, 2008, Adam Schulman p.17.

Philosophy publications and in particular healthcare philosophy papers which include the term dignity as a major discussion point are numerous. Indeed, dignity has become something of an evergreen topic, seemingly a perpetual theme in writings on human rights and health care, offering an opportunity for philosophers and others to exercise ingenuity. Yet, it is not unreasonable to ask whether any of these texts significantly advances our understanding of the concept of human dignity itself or crucially, influences its practical application. In the United States, the President's Council on Bioethics, an eminent body of 17 experts in the field deliberated on dignity over many years, spurred on by rapid developments in the fields of genetics, bioethics and medical innovation. The associated publication Essays Commissioned by the President's Council on Bioethics (2008), some 550 pages long and containing 20 essays written by members of the commission and by others, provides a comprehensive summary of many key aspects though it is clear that there is not an overwhelming

\section{Badcott $(\square)$}

Centre for Applied Ethics, Cardiff University, Humanities Building, Colum Drive, Cardiff CF10 3EU, UK

e-mail: badcottd@cf.ac.uk

C. Leget

Department Ethics of Care, University of Humanistic Studies, Utrecht, The Netherlands consensus regarding the concept itself or indeed its relevance. It seems that if complete agreement or at least substantial accord is what is required, then the search may prove rather futile.

At the same time, the irony is that many if not most members of the general public possess an immediate and clear intuitive understanding of what they perceive to be flagrant infringements of respect for human dignity in health care. Similarly, press reports on aspects of manipulation of fundamental human genetic material, for instance those questioning the use of stem cells or whether "three-parent IVF" should be permitted, tend to stimulate real public concern on either religious grounds or for not being natural. Whilst the term dignity itself might not always feature, it appears at least implicitly to be the nature of the unease. Such concerns as there are indicate that a respect for human dignity is acquired by most of us, like many other fundamental moral attitudes, as part of the process of growing up and living in a cultural community in which certain standards of behaviour are apparent and imbued. Hence, although there may not be significant public awareness of the theoretical concept of dignity nor relevant underlying principles this might not be a serious problem if intuition is all that is required or could be expected. And indeed educating the public could prove to be both difficult and unnecessary other than clearly setting out relevant factual details.

So, against this background, just who needs a theoretical concept of human dignity and why? It would seem that the importance of maintaining and enhancing academic scholarship in this field is most applicable in assisting healthcare professionals and administrators to fully appreciate the nature and relevance of human dignity in their practice and in particular to identify contexts where failure 
to treat patients with due respect for their dignity might arise. In this respect, patients whose personal awareness and autonomy are diminished through age or compromised by mental health difficulties would seem to be those most vulnerable. It should be obvious however that all patients deserve to be treated with a respect which acknowledges their personal dignity. For this to become just as much the norm as other aspects of treatment in health care requires a search for consistency both in terms of what is understood by the concept and illustrated where possible by appropriate contextual examples. And this is where scholars and practitioners can assist by striving for conceptual clarity and providing valuable illustrations.

This small group of papers, some of which were originally presented at a European Conference on Philosophy of Medicine and Health Care held in Zurich, Switzerland in August 2011 addresses either theoretical or practical issues, and in most cases, combines both. The first three papers presented in this section-by Dan Sulmasy, Carlo Leget and Jeanette Pols - analyse different meanings of versions of the concept of dignity. The last three papers-by Roberto Andorno, Charlotte Delmar and Sabine Goethals e.a.-focus on the role of dignity in bioethics and its relation with other values and concepts.

The paper by Daniel Sulmasy (The Varieties of Dignity: a Logical and Conceptual Analysis) tackles head on, the no small matter of whether in view of the various ways in which the term dignity has been utilised, and the periodic criticism on grounds of ambiguity or even superfluity, its continued use has no assured consistency or ethical value. Sulmasy systematically refutes these charges and advocates a 3-part empirical model in which human dignity comprises a spectrum of intrinsic, attributed, and inflorescent (contributing to human flourishing or conducive to human excellence) components. He argues that the latter two logically and linguistically presuppose intrinsic dignity. It is because all human beings possess a fundamental dignity which has an intrinsic value that is prior to human attribution (the key distinction from most if not all other species), that we can appreciate the day-to-day importance of attributed dignity and inflorescent dignity, neither of which could stand alone. Whilst not explicitly indicated, knowledge of this deceptively simple but powerful relationship may assist health care professionals in understanding why respecting the personal dignity of the patients for whom they care is so vital. And recognition of that personal dignity of the individual is manifest in a carer's awareness and respect for their patient's particular attributed and inflorescent sensitivities.

Taking a different approach, Carlo Leget (Analyzing dignity: a perspective from the ethics of care) identifies three versions of the concept of dignity, two of which are rooted in antiquity. Because of "the illocutionary content (reflecting the narrator's intention) that seems to get lost the more one focuses on the details of dignity talk", Leget's objective is not to make an exhaustive analysis but rather "to abstract from the more detailed discussions and search for a unity underlying the various approaches of dignity". The first of Leget's historic concepts relates to social dignity, a labile, third-party ascription entirely dependent on the opinion of others and roughly paralleling Sulmasy's attributed dignity. Importantly this concept can be expanded to reflect social context. Sometimes known as relational dignity, the broadened concept of social dignity can be tied together with feelings of self-respect which can be either enhanced or undermined. The concept of intrinsic dignity which for Sulmasy is pivotal and has priority also has ancient roots. But Leget questions whether although a seemingly stable and perennial concept it is as vulnerable as any idea and therefore subject to the vagaries of philosophical debate. The final part of Leget's dignity trio is experiential subjective dignity for which the author considers both the social/relational and the intrinsic elements automatically engage. An analysis inspired by Paul Ricoeur's philosophy ("helpful in understanding how different philosophical traditions and perspectives may articulate elements that need to be brought together") enables Leget to conclude the interconnectedness and mutual interdependence of the three versions whilst emphasising that social or relational dignity has both genealogical and systematic precedence. Leget offers these considerations to the ethics of care for further reflection. The ethics of care lends itself to social/relational dignity because of the latter's sensitivity to particular situations or context - complex webs of personal relations - rather than generalizable features.

There can be little doubt that rapid hair loss due to cancer treatment can be a particularly traumatic experience and especially so for women. Such loss, has an impact on the psychological and emotional experience of "looking good", and acute feelings of loss of personal dignity. Jeannette Pols' paper "Through the looking glass: good looks and dignity in care", explores female baldness with direct reference to the concept of dignity. Pols distinguishes two meanings of the concept: (1) Humanitas which relates to being human and having intrinsic dignity, and is foundational in concepts of human rights and juridical principles, and (2) the concept of dignity as expressed in the term dignitas. The latter is interpreted with respect to an individual's "engagement in (the) aesthetic genres of sociality they value". Pols' intention is not only to describe how women suffering hair loss feel about and attempt to accommodate or come to terms with this affliction, but at a more theoretical level to demonstrate and promote the value, particularly in health care, of recognising the aesthetic side of dignity. In addition, Pols argues for the 
reintegration of humanitas and dignitas, off-setting the Kantian separation of ethical judgments based on universal, objective fundamental principles and universal but subjective considerations of aesthetics. There are problems for those women affected in that the way the self looks is evaluated both by the woman herself but importantly, and this is a major concern, by others. This is a frank reminder for healthcare practitioners that: "A dignified individual does not exist in a 'minimal' bare state, but only emerges when it is 'dressed up' with aesthetic values, shaped and provided with 'good looks' and particular habits and relations". It is this subjective dimension of dignitas that recognises individuals have an individual and personal accord with aesthetic values that is lacking in and would benefit from being closely associated with the universal humanitas.

In "The dual role of human dignity in bioethics", Roberto Andorno distinguishes between the "overarching policy principle" of dignity and its application as a foundational and guiding role in bioethics and in its contextspecific exercise in patient care. Andorno emphasises that awareness of the importance of respect for human dignity is most apparent and obvious in cases where it is flouted. Although there is often a tendency to sensationalise, instances of serious disregard which are exposed in the media provide powerful reminders to healthcare professionals and members of the public alike. Furthermore, Andorno suggests that the principle of respect for human dignity serves both to establish minimal standards of treatment, with a prohibition on certain practises (a negative requirement) and impacts on the promotion of, for example, quality of life both domestically and regarding health care (positive requirement). It is in the delivery of health care that respect for dignity is often most noticeably threatened and challenged. All patients are to a greater or lesser extent vulnerable. They are vulnerable to the disease or illness from which they are suffering, but also especially vulnerable because of emotional concerns and an asymmetric power relationship with their carers. As Andorno remarks: "If promotion of patients' dignity has always been a crucial element of the medical profession, it has become especially urgent in the time pressured context of modern health care".

Charlotte Delmar has investigated a somewhat overlooked interface by discussing "The interplay between autonomy and dignity: summarizing patients' voices". The paper draws conclusions from empirical research through qualitative interviews with patients. In recent times, the trend has been to encourage patients to adopt a more active role or even self-management in decisions concerning their treatment in recognition of patient autonomy. But confronting some patients with a range of options to decide on their course of treatment may foster feelings of insecurity or powerlessness in coping, provoking difficulties in the relationship between patient and practitioner, and impacting the former's dignity. Delmar highlights a paucity of studies that examine the meanings and implications of selfmanagement from a patient perspective with even fewer that address concerns with self-management and patient dignity. From her review Delmar concludes that although opportunities for self-determination and exercise of personal autonomy are important considerations in the treatment of patients, undue emphasis on this perspective may adversely impact on patient dignity and impede the patient's need for help. The author suggest that further study concerning patients' preparedness to accept autonomous responsibility, practitioners' recognition that not all patients are disposed and capable of making the most beneficial personal choices concerning their care and a focus on the relationship between autonomy and dignity with "different types of patient" is indicated.

Sabine Goethals, Bernadette Dierckx de Casterle' and Chris Gastmans have also addressed some aspects of the autonomy-dignity interface in their paper: "Nurses' ethical reasoning in cases of physical restraint in acute elderly care: a qualitative study". Preserving a patient's dignity during every aspect of their care and treatment is of fundamental importance, none more so than when it is necessary to restrain a patient to prevent self-harm or harm to others. It is acknowledged that the decision-making process associated with the application of physical restraint is complex and although primarily intended to protect the safety and integrity of the patient and others, must also take account of primary healthcare values such as beneficence and respect for autonomy. The paper is an account of a study undertaken to explore the ethical reasoning of nurses in circumstances requiring physical restraint in acute elderly care, and in particular to identify which ethical values are employed. It is apparent that nurses have to strike a balance and exercise proportionality in their treatment of each individual patient. Results were discussed under 5 headings: protecting the value of physical integrity, avoiding physical adverse effects of physical restraint, protecting the value of psychological integrity, protecting the value of dignity and respect for autonomy, and protecting the value of justice. It was concluded that most nurses had the intention of doing good in their treatment of patients, although for some this was implicitly evident in their reasoning and actions, rather than expressly stated (the authors suggest that this latter might be explained by intuitive and tacit knowledge). Overall, the decision-making process is "morally laden" requiring reflection and balance.

Adam Schulman's perception of dignity as "the essential and inviolable core of our humanity" and Ronald Dworkin's idea of dignity as reflecting the intrinsic value of each human life (Is Democracy Possible Here?, 
Princeton University Press 2006) are important facets of the concept that should be understood and acted on by all healthcare practitioners and indeed, everyone. But such notions are not sufficient to form the basis and understanding of sensitive dignified care. The group of papers in this themed section of the journal though not intentionally integrated provide a valuable collection to assist practitioners in working towards a personal understanding which incorporates their own experiences and enhances and informs their quality of care. Though not specifically mentioned in these texts, most European countries and undoubtedly most of the world is undergoing massive social, cultural and technological change. An ever increasing cultural diversity in our multicultural world will require even more sensitivity in the role of dignity awareness in the delivery of individual health care.

\section{References}

Andorno, Roberto. 2013. The dual role of human dignity in bioethics (this issue).

Delmar, Charlotte. 2013. The interplay between autonomy and dignity: Summarizing patients' voices (this issue).

Goethals, Sabine, Bernadette Dierckx de Casterle', and Chris Gastmans. 2013. Nurses' ethical reasoning in cases of physical restraint in acute elderly care: A qualitative study (this issue).

Leget, Carlo. 2013. Analyzing dignity: A perspective from the ethics of care (this issue).

Pols, Jeannette. 2013. Through the looking glass: Good looks and dignity in care (this issue).

Sulmasy, Daniel. 2013. The varieties of dignity: A logical and conceptual analysis (this issue). 